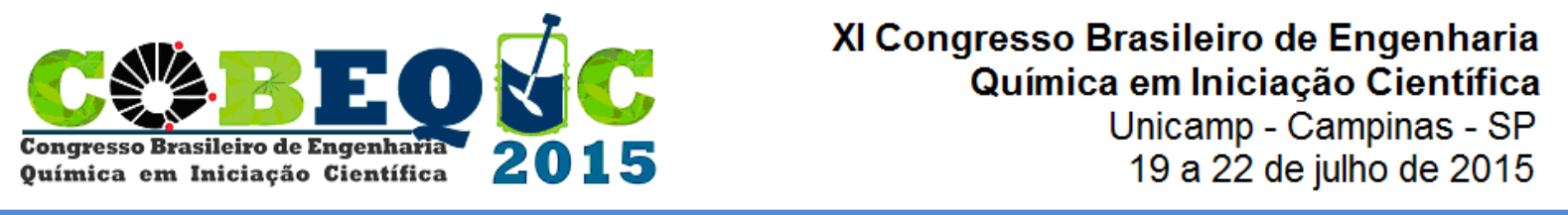

\title{
FILTRAÇÃO DE GASES A ALTAS PRESSÕES: ANÁLISE DAS TORTAS DE FILTRAÇÃO
}

\author{
A. E. de OLIVEIRA ${ }^{1}$, M. L. AGUIAR ${ }^{1}$ \\ ${ }^{1}$ Universidade Federal de São Carlos, Departamento de Engenharia Química \\ E-mail para contato: mlaguiar@ufscar.br
}

\begin{abstract}
RESUMO - O objetivo do presente trabalho foi estudar o comportamento do meio filtrante de celulose no processo de filtração de gases a diferentes pressões, avaliando a queda de pressão no filtro e a porosidade da torta de filtração formada. Os dados obtidos foram comparados com dados existentes na literatura. Concluiu-se que o aumento da pressão ocasionou menor resistência do filtro ao escoamento do gás e consequente menor queda de pressão no filtro. O aumento de pressão também provocou maior retenção de material particulado para uma mesma perda de carga. Também foi concluído que a diminuição do diâmetro volumétrico da partícula ocasionou maior ocorrência de filtração interna sob diferentes pressões do gás.
\end{abstract}

\section{INTRODUÇÃO}

O processo de filtração consiste em uma separação física envolvendo um fluido transportador e componentes particulados ou gasosos presentes no mesmo. Na filtração de agentes particulados, é necessário haver uma velocidade relativa entre a partícula e o corpo de coleta. A captura da partícula ocorre quando há a colisão e consequente aderência da partícula ao corpo de coleta, não se tratando, portanto, de simples peneiração, na qual apenas partículas de tamanho maior que o tamanho dos poros do meio filtrante ficam nele retidas. (DULLIEN, 1989; HINDS, 1999; RICCO JUNIOR, 2002).

Tanabe (2011) avaliou a influência da pressão do gás nas condições de operação da filtração de gás natural utilizando meios filtrantes de poliéster, polipropileno, celulose e malha metlálica e concluiu que, a uma mesma perda de carga, o aumento da pressão possuiu contribuição positiva para a capacidade de filtração (avaliando-se em termos de vazão mássica de gás), aumentando as porosidades das tortas de filtração e a eficiência de coleta de partículas menores que $5 \mu \mathrm{m}$ e diminuindo a resistência específica da torta. Também constatou que, para uma mesma massa de pó depositada, a maior pressão do sistema apresentou a menor perda de carga.

\section{OBJETIVO}

O objetivo deste trabalho foi avaliar o desempenho do meio filtrante de celulose sob diferentes pressões de gás em termos da queda de pressão no meio filtrante e da porosidade da torta de filtração formada. Tais parâmetros são pouco estudados e trabalhos envolvendo a avaliação dessas variáveis são escassos na literatura. Essa pesquisa pretendeu elucidar a 


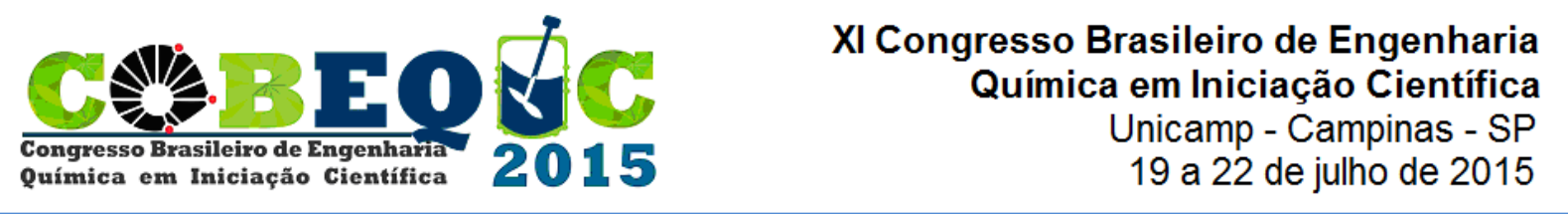

importância da pressão do gás na operação de filtração e apontar caminhos para pesquisas futuras em prol ao desenvolvimento do processo de limpeza de gases.

\section{METODOLOGIA}

O equipamento utilizado nos ensaios de filtração era constituído por um alimentador de pó do tipo rosca (para simular os resíduos sólidos no gás), um compressor de alta pressão ligado a três filtros (para remoção de qualquer tipo de impurezas no ar), um secador (com o intuito de desumidificar o ar), um filtro absoluto, válvulas rotativas e manômetros, um suporte para o meio filtrante e um contador de partículas acoplado a um sistema de aquisição de dados. A Figura 1 consiste na representação do equipamento utilizado para a execução deste trabalho.

Figura 1: Ilustração da bancada de filtração.

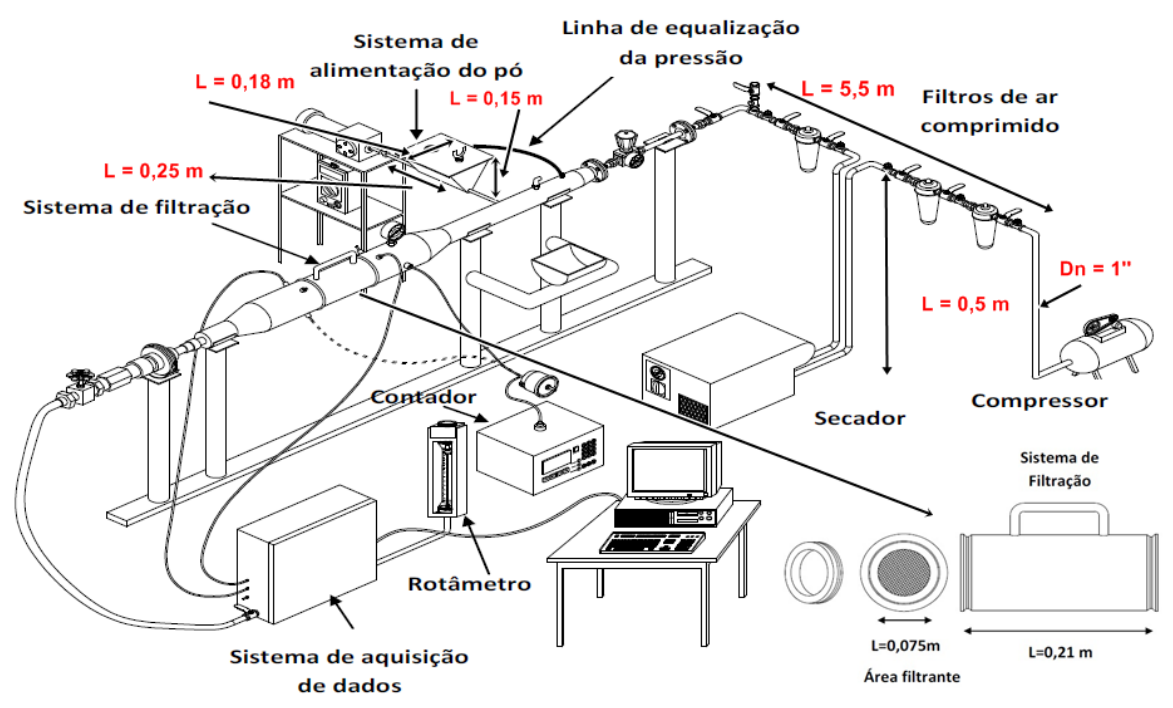

Fonte: Tanabe (2011).

O meio filtrante utilizado, idêntico ao utilizado por Tanabe (2011), era constituído de celulose (gramatura $150 \mathrm{~g} / \mathrm{cm}^{2}$, espessura $0,45 \mathrm{~mm}$, diâmetro das fibras $22 \mu \mathrm{m}$, porosidade volumétrica 0,78 e área de filtração $44 \mathrm{~cm}^{2}$ ).

Para a realização dos testes de permeabilidade, ar comprimido isento de pó atravessava o sistema de filtração a diferentes vazões volumétricas, entre 0 e $85 \mathrm{~L} / \mathrm{min}$ sob pressões de 1,0 a 5,0 bar. Dados de queda de pressão foram mensurados por meio de um sistema de aquisição de dados. Com o auxílio de uma software de cálculos, os dados de queda de pressão foram plotados em função da velocidade superficial do gás, obtida por meio da vazão volumétrica aferida e da área de seção transversal do meio filtrante. Para as pressões de 2,0 a 5,0 bar entretanto, foi necessário utilizar a equação geral dos gases ideais para converter a velocidade do gás, dada pela Equação 1:

$$
v_{S}(T, P)=v_{s 0} \frac{T P R}{T_{R} P}
$$


O método dos mínimos quadrados foi utilizado nas curvas obtidas para a determinação das constantes de permeabilidade da equação modificada de Forchheimer, fornecida pela Equação 2:

$$
\frac{P_{i}^{2}-P_{o}^{2}}{2 P_{o} L}=\frac{\mu}{k_{1}} v_{s}+\frac{\rho}{k_{2}} v_{s}^{2}
$$

Nos testes com pó, foi fixada uma velocidade superficial do gás de $0,049 \mathrm{~m} / \mathrm{s}$, utilizandose a Equação 1 para ajustar as vazões volumétricas a cada mudança de pressão. Foram estudadas as pressões de 2,0, 3,0 e 4,0 bar, utilizando pó de talco como agente particulado e ar comprimido como fluido transportador. Foi estipulada uma perda de carga máxima de $700 \mathrm{~Pa}$ e o tempo de filtração foi aferido com o auxílio de um cronômetro. A queda de pressão foi aferida por meio de um sistema de aquisição de dados. A massa de pó coletada, por sua vez, foi obtida aferindo a massa do meio filtrante antes e após cada ensaio. Com o auxílio de um software de cálculos, os dados de queda de pressão foram plotados em função da massa de pó depositada no filtro por unidade de área. Utilizando o método dos mínimos quadrados apenas na faixa linear das curvas e a Equação 2, foi calculada a resistência específica da torta de filtração para cada pressão avaliada a partir da Equação 3:

$$
\Delta P=K W v_{s}
$$

\section{APRESENTAÇÃO E DISCUSSÃO DOS RESULTADOS}

A partir dos ensaios de permeabilidade, foram obtidos dados de queda de pressão em função da velocidade superficial do gás. Então, foram plotados gráficos relacionando ambas as variáveis e, utilizando o método dos mínimos quadrados, as curvas obtidas foram ajustadas por um modelo quadrático. A queda de pressão, no entanto, foi dada pela primeira parcela da Equação 2, relacionando as pressões de entrada e saída e a espessura do meio filtrante. As curvas experimentais e de ajuste, juntamente às equações de ajuste de cada curva, estão exibidas na Figura 1.

Figura 1 - Curvas de queda de pressão em função da velocidade superficial.

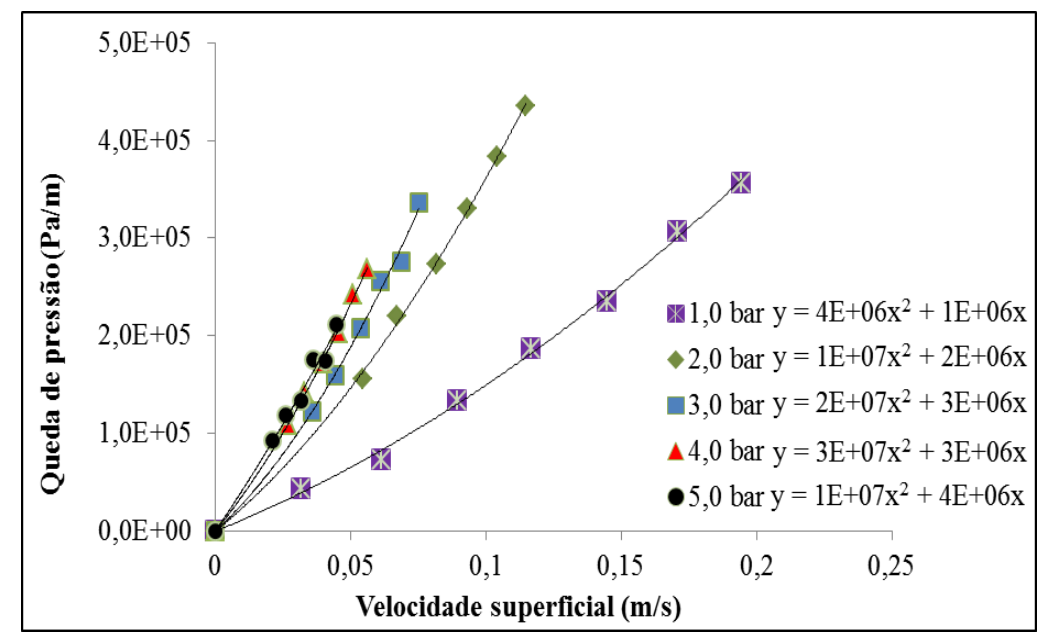


A partir da análise das curvas da Figura 1, verifica-se que, para todas as pressões utilizadas nos testes, o aumento da velocidade superficial aumentou a queda de pressão no meio filtrante. De acordo com Tanabe (2011), esse aumento é devido às contribuições dos termos inercial e viscoso da equação de Forchheimer (Equação 2): o termo linear das curvas representa a queda de pressão devida ao atrito entre as camadas do fluido, quando a baixas velocidades. Com o aumento da velocidade, a mudança de trajetória do gás e suas consequentes alterações da direção de movimento e aceleração provocam o aumento não linear da perda de carga. Também pode ser observado que, com o aumento da pressão do sistema, a uma mesma velocidade superficial de gás, ocorre o aumento da queda de pressão no meio filtrante. Contudo, a taxa de aumento é decrescente com o aumento da pressão (a diferença entre as curvas de 1,0 e 2,0 bar é mais acentuada que entre as curvas de 2,0 e 3,0 bar e assim sucessivamente, de forma que a diferença entre as curvas de 4,0 e 5,0 bar é quase imperceptível graficamente).

A partir dos ensaios de filtração de ar contaminado com pó de talco, os dados de queda de pressão obtidos foram plotados em função das massas de pó coletado no filtro por unidade de área do meio filtrante. Também foram plotadas curvas de queda de pressão em função da massa de pó por unidade de área obtidas por Tanabe (2011), como mostrado na Figura 2.

Figura 2 - Queda de pressão em função da massa de pó coletada por unidade de área: comparação com resultados de Tanabe (2011).

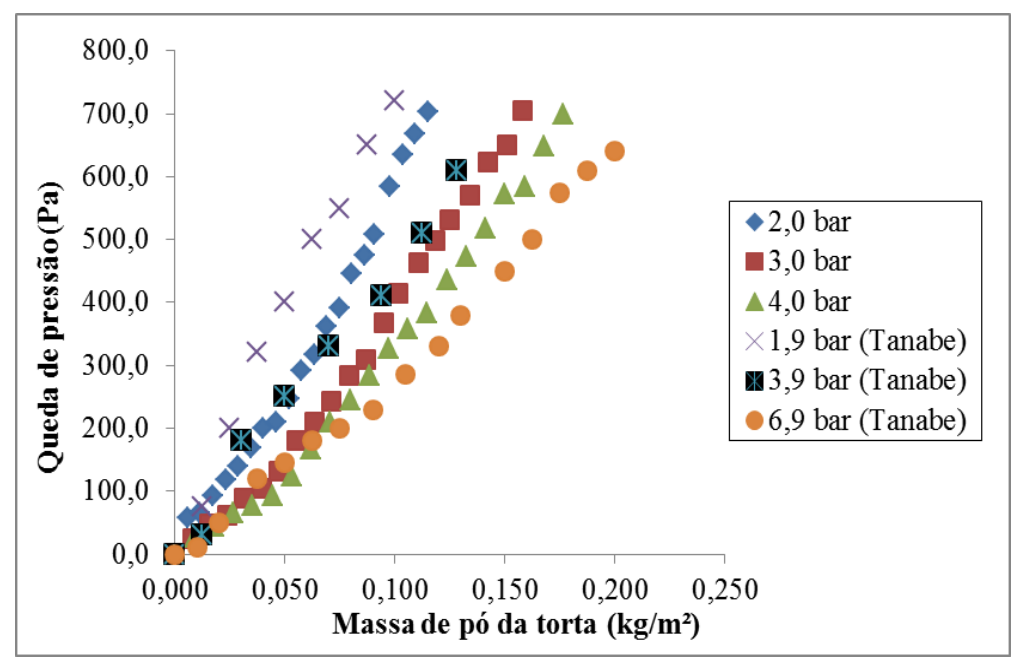

Como pode ser observado pela Figura 2, fixada uma determinada massa de pó, o aumento da pressão do sistema conduziu a uma menor perda de carga no meio filtrante. Analogamente, para uma mesma perda de carga, o aumento da pressão provocou aumento da massa de pó na torta de filtração.

Das curvas, é possível notar que, para os valores de pressão de gás similares de ambos autores (1,9 e 2,0 bar; 3,9 e 4,0 bar), a queda de pressão foi maior para o estudo realizado com rocha fosfática do que aquele realizado com pó de talco, dada mesma massa de pó. Além disso, a parte inicial das curvas de filtração (correspondente à filtração interna) exibe maior discrepância do que o restante das mesmas (filtração devido à formação da torta) comparando curvas de pressões de gás similares. Visto que as condições de operação foram diferentes, é possível que ao menos dois principais fatores tenham influenciado a diferença nas curvas de 
perda de carga. Primeiramente, a pressão de gás utilizada possui diferença de 0,1 bar entre as curvas experimentais comparadas (entre 1,9 e 2,0 bar e entre 3,9 e 4,0 bar) e, como discutido previamente, a pressão é uma variável que interfere na queda de pressão da torta, a uma mesma massa de pó retido. Tal diferença, contudo, foi menor para pressão mais alta, em detrimento da pressão mais baixa, cuja discrepância de resultados é saliente. Outro fator a ser considerado é a diferença de diâmetro volumétrico médio das partículas: enquanto o pó de rocha fosfática possuía $10,5 \mu \mathrm{m}$, o pó de talco possuía $23,67 \mu \mathrm{m}$ de diâmetro volumétrico médio. Assim, as partículas de rocha fosfática, de menor diâmetro volumétrico médio, possuíram menores dificuldades em adentrar os poros do meio filtrante, resultando em maior período de filtração interna (observa-se que, nas curvas de filtração de rocha fosfática, o trecho não linear correspondente à filtração interna - é mais acentuado que nas curvas de filtração de talco e é justamente nesse trecho que a divergência das curvas é mais acentuada).

Das curvas obtidas de perda de carga em função da vazão mássica, foi realizado um ajuste linear em cada curva para a obtenção da resistência específica da torta $(K)$ a partir da Equação 3. Os dados de $\mathrm{K}$ para cada curva foram, então, plotados em um gráfico em função da pressão do gás, como exibido na Figura 3.

Figura 3 - Resistência específica das tortas de filtração de talco.

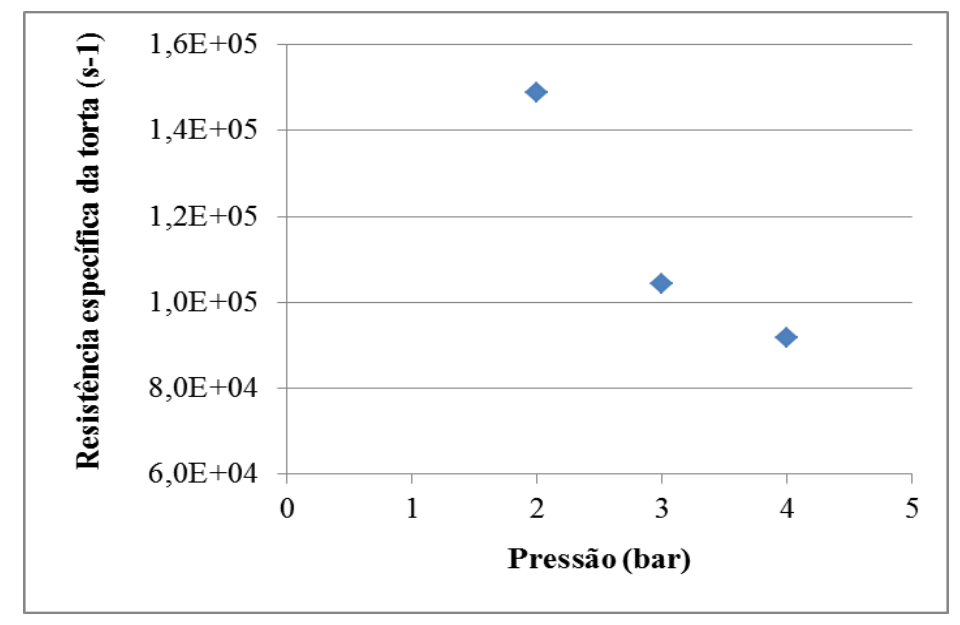

Nota-se, pela Figura 3, a relação inversamente proporcional entre a resistência específica da torta de filtração e a pressão. Assim como afirmou Tanabe (2011), isto significa que as tortas de filtração tendem a se tornar menos resistentes ao escoamento de gás com o aumento da pressão do sistema, resultando em menores perdas de carga para uma mesma massa de pó.

\section{CONCLUSÃO}

Nos testes de permeabilidade do meio filtrante, o acréscimo da velocidade superficial provocou o aumento da queda de pressão do mesmo. Da mesma forma, o aumento da pressão do gás influenciou diretamente a queda de pressão no meio filtrante. Entretanto, a taxa de aumento foi decrescente com o aumento da pressão, ou seja, a uma mesma velocidade superficial de gás, a queda de pressão aumentou menos conforme aumentou-se a pressão do sistema. 
Nos testes de filtração, o aumento da pressão do gás ocasionou menor queda de pressão no filtro. De forma análoga, para uma determinada perda de carga, o aumento de pressão contribuiu para o aumento da massa de pó coletado. Características físicas do material que constituía as partículas poluentes, como o diâmetro volumétrico médio, influenciaram na filtração, provocando maior ocorrência de filtração interna para partículas menores sob diferentes pressões de gás. A resistência específica da torta, K, apresentou relação inversamente proporcional com a pressão, ou seja, o aumento da pressão tornou a torta de filtração menos resistente ao escoamento de gás. Esse fenômeno pode justificar menores quedas de pressão com o aumento da pressão.

\section{NOMENCLATURA}

$K$ - resistência específica da torta $\left(\mathrm{s}^{-1}\right)$

$k_{1}$ - coeficiente de permeabilidade darciana $\left(\mathrm{m}^{2}\right)$

$k_{2}$ - coeficiente de permeabilidade não-darciana (m)

$L$ - espessura do meio filtrante $(\mathrm{m})$

$P$ - pressão do gás $\left(\mathrm{N} / \mathrm{m}^{2}\right)$

$P_{i}$ - pressão de entrada do gás no filtro $\left(\mathrm{N} / \mathrm{m}^{2}\right)$

$P_{o}$ - pressão de saída do gás no filtro $\left(\mathrm{N} / \mathrm{m}^{2}\right)$

$P_{R}$ - pressão do gás de referência $\left(\mathrm{N} / \mathrm{m}^{2}\right)$

$T$ - temperatura do gás $(\mathrm{K})$

$T_{R}$ - temperatura do gás de referência $(\mathrm{K})$

$v_{s}$ - velocidade superficial do gás nas condições de interesse $(\mathrm{m} / \mathrm{s})$

$v_{s 0}$ - velocidade superficial do gás nas condições de referência $(\mathrm{m} / \mathrm{s})$

$W$ - massa de torta por unidade de área $\left(\mathrm{kg} / \mathrm{m}^{2}\right)$

- viscosidade do gás $\left(\mathrm{m}^{2} / \mathrm{s}\right)$

$\rho$ - densidade do gás $\left(\mathrm{kg} / \mathrm{m}^{3}\right)$

\section{REFERÊNCIAS}

DULLIEN, F. A. L. Aerodynamic capture of particles. In: gas cleaning. London: Academic Press, 1989. p. $97-121$.

. Introduction to industrial

HINDS, W. C. Aerosol Technology. 2nd. ed. New York: Wiley Interscience, 1999.

RICCO JUNIOR, E. Lavador de gás tipo Venturi. Meio Filtrante, [s.1.], ano V, n. 25, mar./abr. 2007. Disponível em:

$<$ http://www.meiofiltrante.com.br/materias.asp?action=detalhe\&id=285>. Acesso em: 27 nov. 2012.

TANABE, E. H. Desempenho de meios filtrantes na filtração de gases a altas pressões. 2011, 150 f. Tese de Doutorado. Departamento de Engenharia Química, Universidade Federal de São Carlos, São Carlos, 2011. 